\title{
Towards a Knowledge Graph-Based Explainable Decision Support System in Healthcare
}

\author{
Enayat RAJABI ${ }^{\mathrm{a}, 1}$ and Kobra ETMINANI ${ }^{\mathrm{b}}$ \\ ${ }^{a}$ Cape Breton University, Sydney, NS, Canada \\ ${ }^{\mathrm{b}}$ Center for Applied Intelligent Systems Research (CAISR), \\ Halmstad University, Sweden
}

\begin{abstract}
The decisions derived from AI-based clinical decision support systems should be explainable and transparent so that the healthcare professionals can understand the rationale behind the predictions. To improve the explanations, knowledge graphs are a well-suited choice to be integrated into eXplainable AI. In this paper, we introduce a knowledge graph-based explainable framework for AIbased clinical decision support systems to increase their level of explainability.
\end{abstract}

Keywords. Explainable AI, Knowledge Graph, Clinical decision support system

\section{Introduction}

Artificial Intelligence (AI) is expected to be one of the central areas contributing to the next generation of clinical decision support systems (CDSS). One challenge that has hampered pervasive AI in healthcare is inscrutability. The conclusions and advice derived from a healthcare system must be explainable, and interpretability is considered being a keystone in this transition [1]. EXplainable AI (XAI) approaches assist and foster the AI adoption into clinical settings via building trust and bring transparency to the traditional "black box" models. Most XAI approaches developed in this field require a high level of technical expertise to manipulate algorithms and understanding this mathematical scaffolding is not enough to get insights into internal working models. One approach to increase the explainability of AI-based models in healthcare is leveraging knowledge graphs (KG) and semantic technologies, as they improve explanations for non-technical users who do not have skills in AI technologies. This paper intends to shed more lights on the use of KGs in an explainable CDSS and introduces a framework to leverage KGs in various components of such systems.

\section{Method}

Figure 1 depicts our proposed approach wherein KGs play a central role in different components of a CDSS, as they increase the level of explainability of outputs.

\footnotetext{
${ }^{1}$ Corresponding Author: Enayat Rajabi, 1250 Grand Lake Rd, Sydney, NS B1P 6L2, Canada; E-mail: enayat_rajabi@cbu.ca.
} 
Enriching metadata extracted from medical images with more semantics and meanings, learning input representations of patient data in a neural-network system, providing drug-drug interaction in a medicine recommendation system, and supporting doctors' questions by providing question answering systems over KGs are some activities that use semantics and KGs in a CDSS. Leveraging KGs in different layers of this system substantiates the results with further evidence that acts as an explanation for doctors and healthcare decision makers to make better clinical decisions benefiting both patients and caregivers. As an example, a deep learning system can use the semantic layer of this framework to create class expressions that act as explanations for an image classification problem.

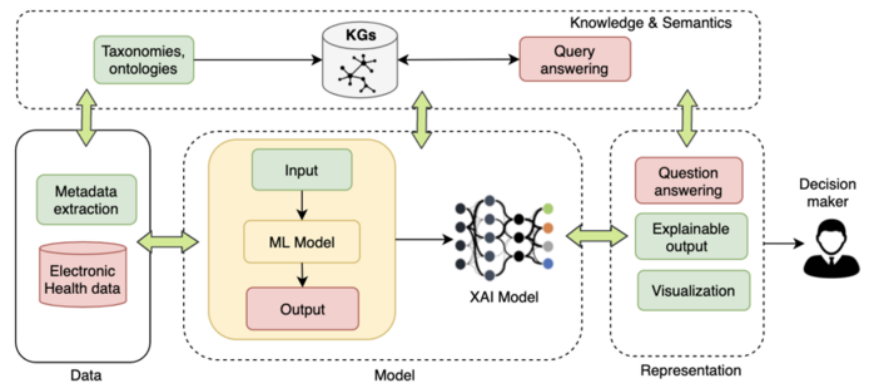

Figure 1. A KG-based explainable clinical decision support system.

\section{Results and Discussion}

KGs are naturally built to be explainable. Existing medical KGs and ontologies (e.g., HG, ICD, UMLS, OGMS) or the other local constructed KGs, can be used in XAI applications to improve overall understandability of complex AI algorithms [2]. As an example, DoctorXAI was proposed as a post-hoc model-agnostic explainable technique focused on local explanations behind any classifier [3] using ICD-9. Healthcare systems often involve diagnosis prediction and use taxonomical KGs to improve the interpretability and understandability of their XAI models.

\section{Conclusion}

We introduced a KG-based explainable framework for AI-based CDSS. The framework can be used as a guideline to design a new CDSS or integrate the KG-based XAI into an existing healthcare system.

\section{References}

[1] Barh D. Artificial Intelligence in Precision Health: From Concept to Applications. Academic Press; 2020.

[2] Seeliger A, Pfaff M, Krcmar H. Semantic Web Technologies for Explainable Machine Learning Models: A Literature Review. 2019.

[3] Panigutti C, Perotti A, Pedreschi D. Doctor XAI: an ontology-based approach to black-box sequential data classification explanations. In Proceedings of the 2020 conference on fairness, accountability, and transparency 2020 Jan 27. p. 629-639. 